\title{
Pannexin-1 silencing inhibits the proliferation of U87-MG cells
}

\author{
LI WEI $^{1,2}$, XIAOBO YANG $^{1,2}$, XIAOHONG SHI $^{3}$ and YINGHUI CHEN ${ }^{1,2}$ \\ ${ }^{1}$ Department of Neurology, Jinshan Hospital, Fudan University, Shanghai 201508; \\ ${ }^{2}$ Department of Neurology, Shanghai Medical College, Fudan University, Shanghai 200032, \\ ${ }^{3}$ Department of Endocrinology, Jinshan Hospital, Fudan University, Shanghai 201508, P.R. China
}

Received December 15, 2013; Accepted July 23, 2014

DOI: $10.3892 / \mathrm{mmr} .2015 .3169$

\begin{abstract}
Pannexin-1 (Panx-1) is abundantly expressed in vertebrates and has been shown to assemble into high-conductance single-membrane channels, which are permeable to large molecules and regulate cellular function. However, the association between Panx-1 and astrocyte proliferation is poorly understood. This study provides evidence for a difference in cell proliferation between wild-type and Panx-1-knockdown cells. Proliferation of the U87-MG malignant glioma cell line was reduced following transfection with Panx-1-short interfering RNA. In addition, treatment with the Panx-1 activator, adenosine triphosphate, significantly reduced cell proliferation at $48 \mathrm{~h}$ in Panx-1-knockdown cells compared with wild type cells. In conclusion, on the basis of the present findings, Panx-1 is likely to be important in the regulation of U87-MG cell proliferation. This provides further support for the hypothesis that there is a correlation between Panx-1 expression and U87-MG cell proliferation.
\end{abstract}

\section{Introduction}

There is accumulating evidence that intercellular communication is essential in the spread of signals between cells. It is involved in a number of physiological (rhythmic electrical activity) and pathological (schizophrenic disorders and inflammatory responses) processes. Gap junctions are the only junctional structures that are conserved in all multicellular organisms, and not only connect neighboring cells but also permit the exchange of molecules between the cytoplasm and the extracellular space (1). Pannexins (Panxs), which were described as a new member of

Correspondence to: Mr. Yinghui Chen, Department of Neurology, Jinshan Hospital, Fudan University, 1508 Longhang Road, Shanghai 201508, P.R. China

E-mail: yinghuichen@fudan.edu.cn

Mrs. Xiaohong Shi, Department of Endocrinology, Jinshan Hospital, Fudan University, 1508 Longhang Road, Shanghai 201508, P.R. China

E-mail: shixh80301@163.com

Key words: Pannexin-1, glioma cell, astrocytes, cell proliferation, RNA interference the family of gap junctions in 2000, are permeable to relatively large molecules, including adenosine triphosphate (ATP) (2-5). ATP in turn binds to metabotropic P2Y receptors, leading to $\mathrm{Ca}^{2+}$ release from intracellular stores. $\mathrm{Ca}^{2+}$ then activates Panx-1 hemichannels again inducing the release of ATP. This is termed ATP-induced ATP release. By facilitating ATP-induced ATP release and $\mathrm{Ca}^{2+}$-wave propagation, Panx-1 has an important function in a number of cellular processes $(3,6,7)$. For instance, Panx-1 mediates neuronal death, affects keratinocyte differentiation and regulates the proliferation of human subcutaneous fibroblasts, and neural stem and progenitor cells (8-11).

Astrocytes are the most abundant non-neuronal cells in the central nervous system and are crucial to a number of physiological and pathophysiological processes. Glioma is the most common form of malignant brain tumor, and is associated with a poor prognosis. A study has shown that Panx-1 acts as a tumor-suppressor protein in the development of C6 gliomas (12). However, the association between Panx-1 and astrocyte proliferation remains poorly understood. The current study examined the effect of Panx-1 on the proliferation of U87-MG malignant glioma cells as well as examining the effects of ATP on cell proliferation and apoptosis in U87-MG cells that were or were not expressing Panx-1. This was achieved through the use of immunohistochemistry, short interfering RNA (siRNA) transfection and Cell Counting kit-8 (CCK-8) assays. Proliferating cell nuclear antigen (PCNA) was used to identify proliferating cells.

\section{Materials and methods}

Cell lines and cell culture. The U87 human malignant glioma cell line (U87-MG) were obtained from the Chinese Academy of Sciences (Shanghai, China). The cells were cultured in Dulbecco's modified Eagle's medium (HyClone, Logan, UT, USA) containing a high concentration of glucose, supplemented with $10 \%$ fetal bovine serum (FBS; Gibco Life Technologies, Grand Island, NY, USA) and a mixture of antibiotics-antimycotics (HyClone) in an atmosphere of $5 \% \mathrm{CO}_{2} / 95 \%$ air at $37^{\circ} \mathrm{C}$. The cells were passaged every $4-5$ days to maintain exponential growth and were not used beyond the twentieth passage.

Immunocytochemistry. U87-MG cells grown on coverslips were fixed with $4 \%$ paraformaldehyde for $30 \mathrm{~min}$, washed several times with 0.1 M phosphate-buffered saline ( $\mathrm{pH} 7.4)$ and then incubated for $20 \mathrm{~min}$ in $0.3 \% \mathrm{H}_{2} \mathrm{O}_{2}$, which had been 
diluted in methanol to quench the endogenous peroxidase activity. Coverslips were blocked with $10 \%$ normal goat serum for $30 \mathrm{~min}$. A rabbit polyclonal anti-Panx-1 antibody (Abcam, Cambridge, UK) was diluted to 1:1,000. The secondary antibody was goat anti-rabbit immunoglonulin $\mathrm{G}$ conjugated to horseradish peroxidase (HRP) for detection (Changdao-Bio, China). Subsequently, 3,3'-diaminobenzidine (Maixin-Bio, Fuzhou, China) was used to develop the color reaction. Finally, sections were counterstained with hematoxylin, dehydrated and coverslipped. Immunostaining was also performed in samples prepared without the primary antibody as a negative control.

siRNA transfection. Cell transfection was conducted with Lipofectamine $2000^{\mathrm{TM}}$ reagent (Invitrogen Life Technologies, Carslbad, CA, USA), according to the manufacturer's instructions. Briefly, U87-MG cells were seeded in 6-well plates. Once the cells were 50-80\% confluent, the appropriate treatments were applied. For siRNA experiments, media lacking antibiotics was used to improve transfection efficiency. U87-MG cells were transfected with $75 \mu \mathrm{M}$ Panx-1-specific siRNA (Santa Cruz Biotechnology, Inc., Santa Cruz, CA, USA) using the Lipofectmine $2000^{\mathrm{TM}}$ reagent. Scrambled control siRNA (Santa Cruz Biotechnology, Inc.) with no homology to any mammalian sequence was used as a negative control. Cells were harvested $48 \mathrm{~h}$ after transfection for analysis.

Reverse transcription-quantitative polymerase chain reaction (RT-qPCR). Total cellular RNA was extracted with TRIzol reagent (Invitrogen Life Technologies) according to the manufacturer's instructions. The quality of the RNA was confirmed using formaldehyde-agarose gel electrophoresis. RNA (500 ng) was used to obtained template cDNA using a PrimeScript ${ }^{\mathrm{TM}}$ RT master mix (Takara Bio, Inc., Shiga, Japan) for qPCR using SYBR Premix Ex TaqII on a ABI 7300 system (Takara Bio, Inc.). The following specific PCR primers designed by Bio-TNT (Shanghai, China) were used: Forward: 5'-AAT CTG TGA CTT CTG CGA CAT-3' and reverse: 5'-CCA TTT CCA TTA GGG ACT CAA-3' for Panx-1; forward: 5'-TTA GCT CCA GCG GTG TAA AC-3' and reverse: 5'-CAG CGG TAG GTG TCG AA-3' for PCNA; and forward: 5'-AAGGTGACAGCAGTCGGTT-3' and reverse: 5'-TGTGTGGACTTGGGAGAGG-3' for $\beta$-actin (the reference gene). Samples were run in triplicate and the relative levels of mRNA expression were analyzed relative to $\beta$-actin levels using the comparative cycle $(\mathrm{Ct})$ threshold method. Following PCR amplification, all the samples were verified by $2 \%$ agarose gel electrophoresis.

Western blot analysis. Total cellular proteins were extracted with sodium dodecyl sulfate (SDS) lysis buffer, heated for $5 \mathrm{~min}$ at $99^{\circ} \mathrm{C}$ and then centrifuged for at $16,000 \mathrm{x}$ for $5 \mathrm{~min}$. Protein concentrations were determined using a bicinchoninic acid protein assay kit (Beyotime, Shanghai, China). Samples (20 $\mu \mathrm{g} /$ well) were resolved on a 10\% SDS-polyacrylamide gel electrophoresis gel and then electrophoretically transferred to Immobilon ${ }^{\circledR}$-P membranes (Millipore, Billerica, MA, USA). Pre-stained molecular markers (Fermentas, Pittsburgh, PA, USA) were used as a reference for the molecular weight of the proteins. Membranes were blocked with 5\% non-fat milk in $1 \mathrm{X}$ Tris-buffered saline with Tween-20 for $1 \mathrm{~h}$ and subsequently incubated with either rabbit polyclonal anti-Panx-1 antibody $(1: 1,000)$ or mouse polyclonal anti-PCNA (1:400; Cruz Biotechnology, Inc.) antibodies overnight at $4^{\circ} \mathrm{C}$, prior to incubation with the appropriate HRP-conjugated goat monoclonal anti-rabbit (1:1000; Millipore, Billerica, MA, USA) or goat monoclonal anti-mouse (1:1,000; Millipore, Billerica, MA, USA) secondary antibodies for $1 \mathrm{~h}$ at room temperature. Immunoreactivity was detected by enhanced chemiluminescence detection using the chemiluminescent HRP substrate kit (Millipore). The band densities were quantified with the image-analysis software (Tinon Software, Zhongshan, China). All data were normalized to GAPDH.

Cell proliferation assay. Cells transfected with Panx-1-specific siRNA or scrambled siRNA for $24 \mathrm{~h}$ were seeded on 96 -well plates at a density of 1,000 cells $/ 100 \mu$ l culture medium containing $10 \%$ FBS per well. Cell proliferation was estimated using CCK-8 (Dojindo, Kumamoto, Japan) according to the manufacturer's instructions at 1, 6, 12, 24 and $48 \mathrm{~h}$ after cells were seeded. Briefly $10 \mu \mathrm{l}$ reagent was mixed with $100 \mu$ l culture medium and incubated for 1-3 h in a cell incubator (Lishen, Shanghai, China). Absorbance at $450 \mathrm{~nm}$ was measured using an enzyme-linked analyzer (Biotek Instruments Inc., Winooski, VT, USA). Experiments were repeated five times.

ATP treatment. U87-MG cells were seeded in 96-well plates in five parallel wells and treated with $0.1,1$ and $5 \mu \mathrm{mol} / \mathrm{ml}$ ATP (Sigma-Aldrich, St. Louis, MO, USA). At the predetermined time points, cell proliferation was estimated using a CCK-8 assay. Protein levels of Panx-1 and PCNA were estimated by western blot analysis, as described above.

Statistical analysis. Calculations were performed with GraphPad InStat, Version 5.0 (GraphPad Prism Software, San Diego, CA, USA). Student's two-tailed t-test was utilized for all data analysis and values are expressed as the mean \pm standard error of the mean acquired from at least two independent experiments. $\mathrm{P}<0.05$ was considered to indicate a statistically significant difference.

\section{Results}

Panx-1 expression is increased in mitotic U87-MG cells. The results from the immunocytochemistry experiments showed that Panx-1 protein was located in the cytoplasm. The degree of staining varied between cells. As shown in Fig. 1, Panx-1 staining was predominantly observed in cells in the process of mitosis. Cells at the mitotic phase, with chromosomes arranging in a flower-like ring and arranging at the equator of the spindle, and cells just finishing mitosis were positively immunostained. This suggests that Panx-1 expression may be associated with the proliferation of U87-MG cells.

Panx-1 and PCNA mRNA and protein expression following cell transfection. Based on the immunohistochemical results, cell transfection experiments were conducted. Panx-1-specific siRNA was effective in knocking down endogenous expression of Panx-1 at mRNA (Fig. 2A, 66\%, P<0.01) and protein levels (Fig. 2B, 52.5\%, P<0.05) in U87-MG cells. Knockdown 
A

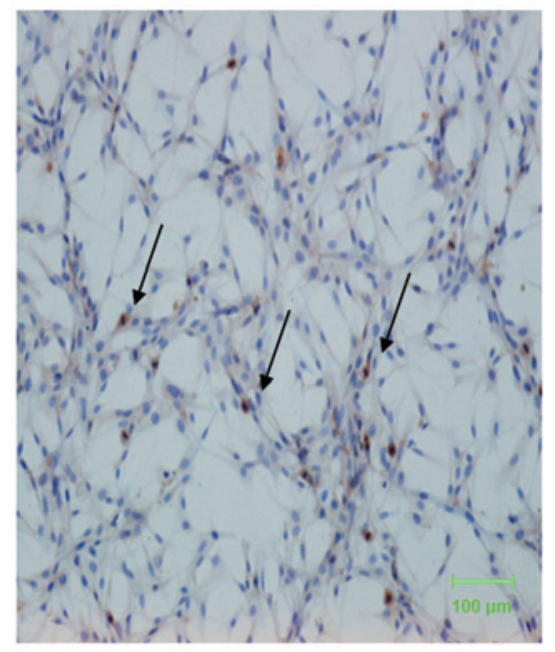

B

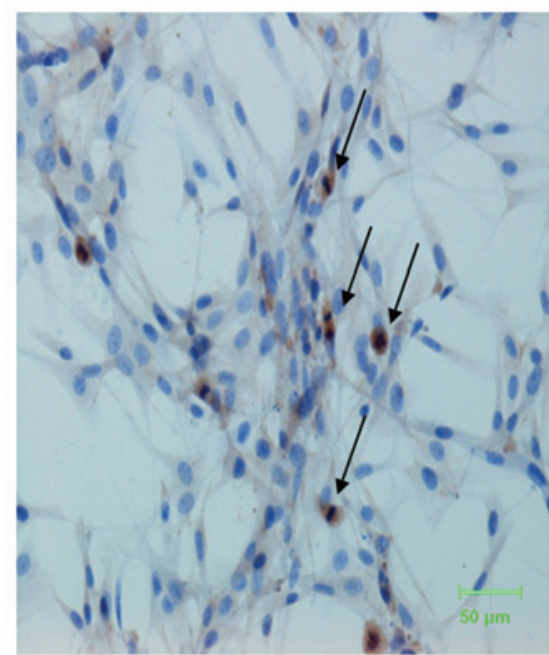

Figure 1. Expression of Panx-1 in U87-MG cells. 3,3'-diaminobenzidine staining was weakly positive in U87-MG cells. However, mitotic cells stained positive for antibodies against human Panx-1. Arrows indicate cells at the mitotic phase, which exhibit positive immunostaining. (A) magnification, x50; scale bar, $100 \mu \mathrm{m}$. (B) Magnification, x200; scale bar, $50 \mu \mathrm{m}$.

A



B

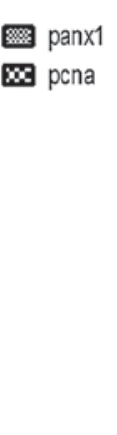

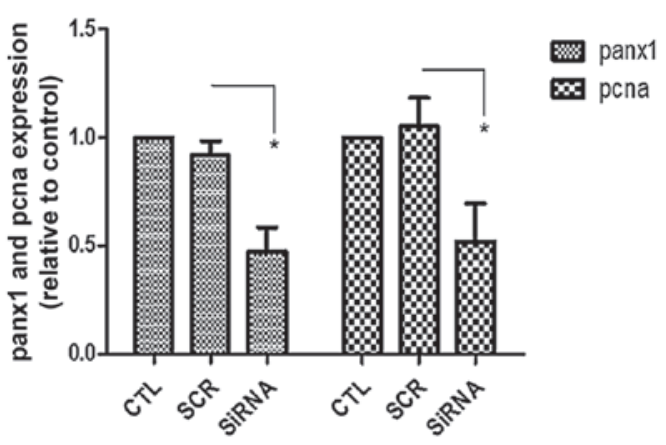

C

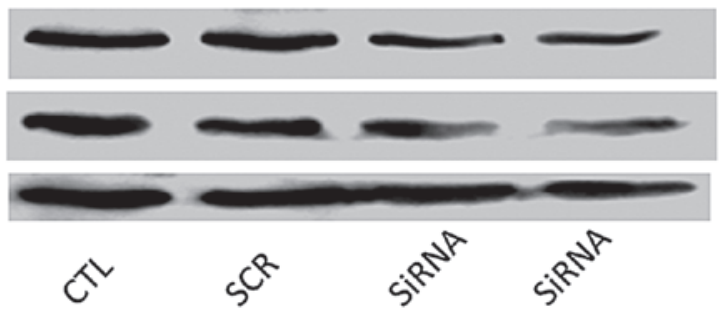

Panx-1 48 kDa

PCNA $36 \mathrm{kDa}$

GAPDH $36 \mathrm{kDa}$

Figure 2. Expression of Panx-1 and PCNA mRNA and protein in U87-MG cells after siRNA transfection (48 h processing time). siRNA specific to Panx-1 or SCR was transfected into U87-MG cells. (A) Levels of Panx-1 and PCNA mRNA after siRNA transfection. (B) Protein expression of Panx-1 and PCNA after siRNA transfection. (C) Western blot showing Panx-1 and PCNA protein downregulation by siRNA transfection. Data are presented as the mean \pm standard error of the mean and were obtained from at least three experiments. The control group contains cells with no treatment in order to confirm Lipofectamine $2000^{\mathrm{TM}}$ had no effect on cell proliferation. ${ }^{*} \mathrm{P}<0.5$ and ${ }^{* *} \mathrm{P}<0.01$. Panx-1, Pannexin-1; PCNA, proliferating cell nuclear antigen; CTL, control; SCR, scrambled short interfering RNA vector; siRNA, short interfering siRNA.

of Panx-1 did not alter PCNA mRNA levels, but western blot analysis revealed that PCNA protein expression in the Panx-1-specific siRNA transfection group was reduced by 47.8\% ( $\mathrm{P}<0.05)$.

Effects of Panx-1 silencing on the proliferation of U87-MG cells. To investigate the role of Panx-1 in the regulation of cell proliferation, the effects of Panx-1-specific siRNA on the proliferation of U87-MG cells were investigated. Cells were transfected either with Panx-1-specific siRNA or a scrambled control siRNA using Lipofectamine 2000 and the proliferation of U87-MG cells was determined using a CCK- 8 assay at various timepoints $(1 \mathrm{~h}$, and $1,2,3$ and 4 days) after cells had been seeded. The results from the CCK-8 assay showed that the optical density (OD) at 450 of the Panx-1-specific siRNA group was decreased significantly compared with the scrambled siRNA control at 1-4 days (Fig. 3). 

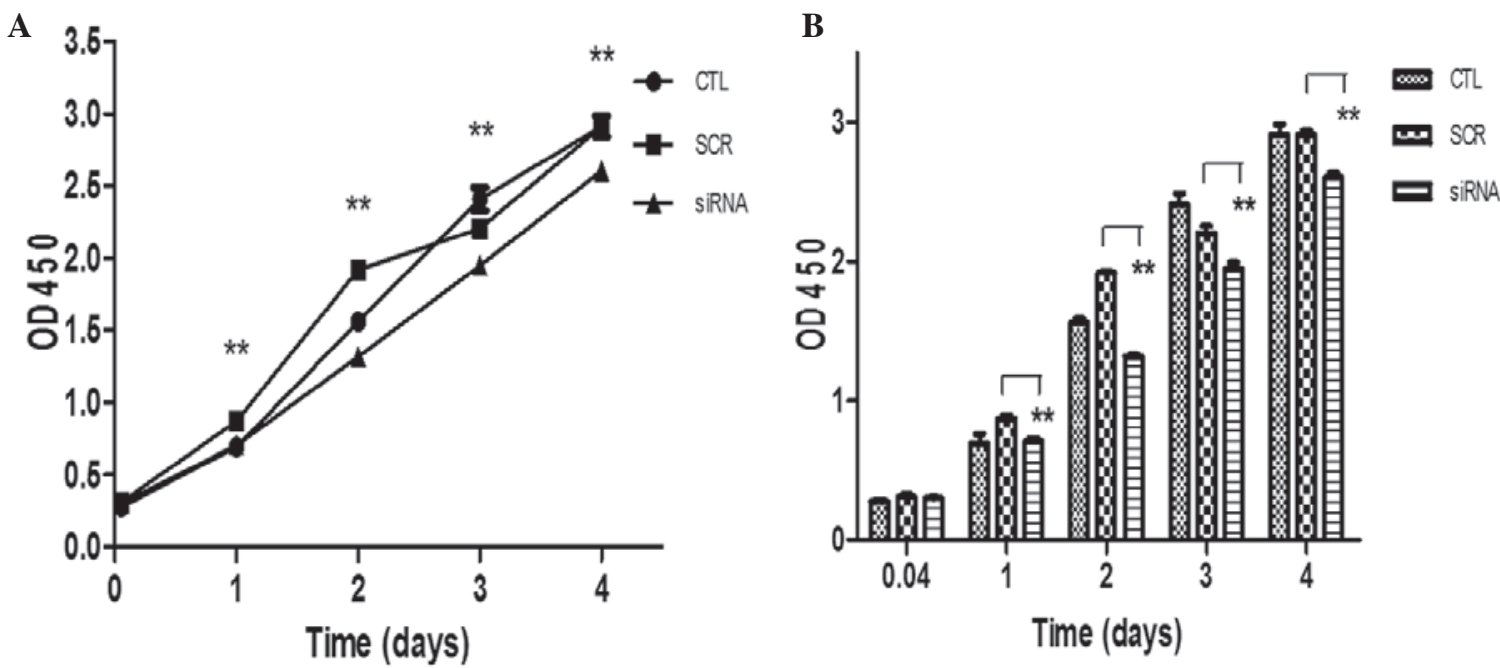

Figure 3. Measurement of cell proliferation of U87-MG cells after Panx-1 silencing using CCK-8. Cells were transfected with siRNA specific to Panx-1 or SCR. (A) Proliferation curve and (B) graph showing the OD 450 after Panx-1 silencing at the processing time of $1 \mathrm{~h}$ and $1,2,3$ and 4 days. ${ }^{* *} \mathrm{P}<0.01$, compared with the SCR group. CCK-8, Cell Counting kit-8; OD 450, optical density absorbance at $450 \mathrm{~nm}$; Panx-1, Pannexin-1; CTL, control; SCR, scrambled short interfering RNA vector; siRNA, short interfering RNA.

A



B

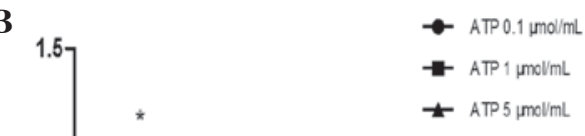

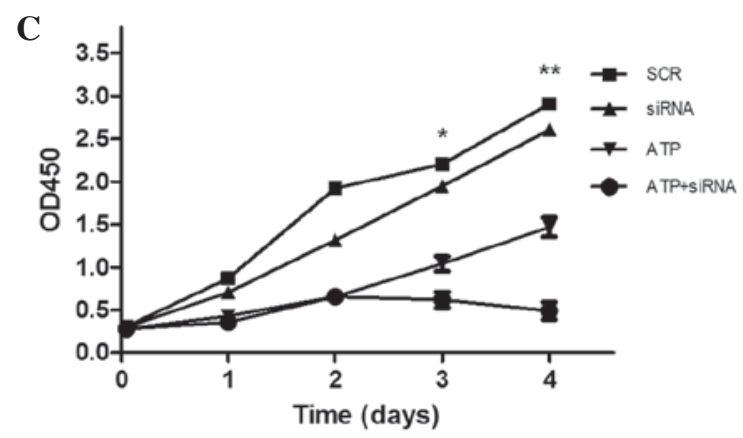

Figure 4. Measurement of cell proliferation and cell viability of U87-MG cells, with the treatment indicated, using CCK-8. (A) Cell proliferation with administration of varying concentrations of ATP. (B) Cell viability with administration of varying concentrations of ATP. * $<0.05$, compared with the untreated cells $(0 \mu \mathrm{mol} / \mathrm{ml})$. (C) Cell proliferation with ATP and Panx-1-siRNA treatment. ${ }^{*} \mathrm{P}<0.05$ and ${ }^{* *} \mathrm{P}<0.01$ for siRNA, vs. ATP+siRNA. OD 450 , optical density at $450 \mathrm{~nm}$; ATP, adenosine triphosphate; SCR, scrambled short interfering RNA vector; siRNA, short interfering RNA.

This indicates that Panx-1 silencing significantly inhibits the proliferation of U87-MG cells.

ATP-induced decrease in cell proliferation is enhanced by Panx-1 silencing. In order to investigate the correlation between Panx-1 and cell proliferation, the effect of varying levels of ATP in Panx-1 knockdown cells was observed. The effects of different concentrations of ATP on cell proliferation and cell viability were examined. As shown in Fig. 4A, each of the three concentrations led to a reduction in U87-MG cell proliferation, and the effect of the $5 \mu \mathrm{mol} / \mathrm{ml}$ dose was statistically significant. It can also be seen from Fig. 4B that $5 \mu \mathrm{mol} / \mathrm{ml}$ ATP led to a significant decrease in cytotoxicity. However, the moderate concentration $(1.0 \mu \mathrm{mol} / \mathrm{ml})$ of ATP 
was the most effective at inhibitong cell proliferation and cell viability at the $6 \mathrm{~h}$ time point. For this reason, $1.0 \mu \mathrm{mol} /$ $\mathrm{ml}$ ATP was selected for the remainder of the cell viability assays. Fig. 4C shows that combining Panx-1 knockdown with the administration of ATP markedly inhibited the proliferation of U87-MG cells compared with either of these factors alone.

\section{Discussion}

Panxs are a novel group of gap junction proteins and have a structure similar to that of connexins (Conxs). Panxs and Conxs are four-pass transmembrane proteins, with intracellular amino-(NH2) and carboxy- $(\mathrm{COOH})$ termini. The Panx family comprises three members: Panx-1, Panx-2 and Panx-3 $(1,2)$. Panx-1 is highly expressed in the central nervous system, while Panx-2 is largely restricted to the brain and Panx-3 is found in the skin, the male reproductive tract of the adult rat, osteoblasts and mature growth plate chondrocytes (13-15). Panx-1 is able to form homomeric and heteromeric channels in combination with Panx-2. It is expressed by several organisms and has been shown to be critical in mediating cell growth $(7-12,16,17)$. However, little is known about the effect of Panx-1 on glioma cell development. The present study reports that Panx-1 expression and U87-MG cell proliferation are closely correlated.

Using immunocytochemistry, it was shown that Panx-1 was expressed in the cytoplasm of U87-MG cells. This was consistent with a prior study that used reverse transcription PCR, but which did not demonstrate the expression of Panx-1 in the U87-MG cells using immunocytochemistry or immunofluorescence (12). However, the results of the current study were different from those of previous studies, which demonstrated that Panx-1 is predominantly located on the cellular membrane in primary cultured astrocytes $(18,19)$. To the best of our knowledge, this is the first study demonstrating the location of Panx-1 in U87-MG cells. In addition, Panx-1 staining was predominantly observed in mitotic cells, which provides support for the hypothesis that Panx-1 may participate in the growth of glioma cells. A knockdown model of Panx-1 was therefore produced in order to test this hypothesis. PCNA, an ancestral nuclear protein involved in DNA replication, has a strong association with cancer transformation (20-22). It was used in the present study as a marker to track cell proliferation during the cell transfection process. It was shown that expression of Panx-1 was silenced by specific siRNAs at the mRNA and protein level. In addition, as shown in Fig. 2, the change in protein levels of Panx-1 was correlated with that observed in PCNA protein levels. However, it is noteworthy that PCNA mRNA levels did not change in the same manner. This may be attributed to different regulatory mechanisms acting on the synthesis and degradation of mRNA and proteins, which affect their quantity (23). This result provided strong evidence that Panx-1 may be involved in the regulation of U87-MG cell proliferation.

Prior studies have shown Panx-1 may be gated by membrane depolarization, mechanical stimulation, extracellular $\mathrm{K}^{+}$, intracellular $\mathrm{Ca}^{2+}$ release and ATP. Furthermore, ATP has been implicated in the regulation of skeletal muscle proliferation, differentiation and regeneration $(24,25)$. It was hypothesized that, as Panx-1 acts as an ATP-releasing channel, Panx-1 knockdown may induce a downregulation of extracellular
ATP concentration. The present study demonstrated that ATP markedly inhibited the proliferation of U87-MG cells, therefore, Panx knockdown may increase cell proliferation. A study conducted in C6 glioma cells provided evidence in support of this theory (12). It revealed that Panx was not expressed in these cells, but that transfection with Panx-1 resulted in suppression of glioma cell growth. The current study used a CCK-8 assay to analyze whether there was a synergistic effect in inhibiting cell proliferation between ATP treatment and Panx-1 silencing. Contrary to the hypothesis that Panx-1 knockdown ultimately leads to an increase in cell proliferation via reducing extracellular ATP, treatment with ATP led to a greater downregulation of cell proliferation in the Panx-1-specific siRNA-transfected U87-MG cells compared with control siRNA-transfected cells, knockdown cells or ATP treated cells alone. This suggests that Panx-1 located in the intracellular space is not able to form an ATP-releasing channel, meaning that Panx-1 silencing had no effect on the extra- and intracellular ATP concentration, and therefore was unable activate the purinergic signaling pathway. However, further research is required to test this hypothesis, including the use of BrdU or EdU incorporation assays or flow cytometry to define progress through the cell cycle.

Glioma cell proliferation and migration are associated with the metastasis of cancer cells. The modulation of cell proliferation is therefore important in cancer biology research. Probenecid, which has been used as an effective clinical drug for the treatment of arthritis, has been shown to be an effective Panx-1 channel blocker (26-28). This may prove to be a novel method of treatment for glioma. However, its nonspecificity not only limits its use as a Panx-1 channel blocker, as it is also used as a multidrug resistance transporter-1 (MRP1) blocker (29-31). As a novel clinical treatment, gene therapy is currently accepted as one of the most promising strategies for cancer therapy. siRNAs have been shown to have effective biomedical genetic-therapy applications for a number of diseases. siRNAs induce sequence-specific gene silencing of target mRNAs and thus alter the expression of molecules involved in tumor development. However, the majority of these studies have been conducted at the cellular level and further studies are required to explore the effects of siRNA interference in vivo (32-36).

\section{Acknowledgements}

This study was supported by grants from the Shanghai Municipal Health Bureau (grant no. 2012-234) to Mr. Yinghui Chen.

\section{References}

1. Barbe MT, Monyer H and Bruzzone R: Cell-cell communication beyond connexins: the pannexin channels. Physiology (Bethesda) 21: 103-114, 2006.

2. Panchin Y, Kelmanson I, Matz M, et al: A ubiquitous family of putative gap junction molecules. Curr Biol 10: R473-R474, 2000.

3. Suadicani SO, Iglesias R, Wang J, et al: ATP signaling is deficient in cultured Pannexin1-null mouse astrocytes. Glia 60: 1106-1116, 2012.

4. Dahl G and Keane RW: Pannexin: from discovery to bedside in

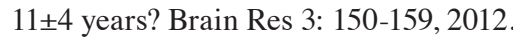

5. Penuela S, Gehi R and Laird DW: The biochemistry and function of pannexin channels. Biochim Biophys Acta 1828: $15-22,2013$. 
6. D'hondt C, Ponsaerts R, De Smedt H, et al: Pannexin channels in ATP release and beyond: An unexpected rendezvous at the endoplasmic reticulum. Cell Signal 23: 305-316, 2011.

7. Gulbransen BD, Bashashati M, Hirota SA, et al: Activation of neuronal P2X7 receptor-pannexin-1 mediates death of enteric neurons during colitis. Nat Med 18: 600-604, 2012.

8. Celetti SJ, Cowan KN, Penuela S, et al: Implications of pannexin 1 and pannexin 3 for keratinocyte differentiation. J Cell Sci 123: 1363-1372, 2010

9. Orellana JA, Froger N, Ezan P, et al: ATP and glutamate released via astroglial connexin 43 hemichannels mediate neuronal death through activation of pannexin 1 hemichannels. J Neurochem 118: 826-840, 2011.

10. Pinheiro AR, Paramos-de-Carvalho D, Certal M, et al: Histamine induces ATP release from human subcutaneous fibroblasts, via pannexin-1 hemichannels, leading to $\mathrm{Ca}^{2+}$ mobilization and cell proliferation. J Biol Chem 288: 27571-27583, 2013.

11. Wicki-Stordeur LE, Dzugalo AD, Swansburg RM, et al Pannexin 1 regulates postnatal neural stem and progenitor cell proliferation. Neural Devel 7: 11, 2012.

12. Lai CP, Bechberger JF, Thompson RJ, et al: Tumor-suppressive effects of pannexin 1 in C6 glioma cells. Cancer Res 67: $1545-1554,2007$.

13. Iwamoto T, Nakamura T, Doyle A, et al: Pannexin 3 regulates intracellular ATP/cAMP levels and promotes chondrocyte differentiation. J Biol Chem 285: 18948-18958, 2010.

14. Bond SR, Lau A, Penuela S, et al: Pannexin 3 is a novel target for Runx2, expressed by osteoblasts and mature growth plate chondrocytes. J Bone Miner Res 26: 2911-2922, 2011.

15. Turmel P, Dufresne J, Hermo L, et al: Characterization of pannexin 1 and pannexin 3 and their regulation by androgens in the male reproductive tract of the adult rat. Mol Reprod Dev 78 : 124-138, 2011

16. Chekeni FB, Elliott MR, Sandilos JK, et al: Pannexin 1 channels mediate 'find-me' signal release and membrane permeability during apoptosis. Nature 467: 863-867, 2010.

17. Tan C, Voss U, Svensson S, et al: High glucose and free fatty acids induce beta cell apoptosis via autocrine effects of ADP acting on the P2Y(13) receptor. Purinergic Signal 9: 67-79, 2013

18. Santiago MF, Veliskova J, Patel NK, et al: Targeting pannexin 1 improves seizure outcome. PLoS One 6: e25178, 2011.

19. Karpuk N, Burkovetskaya M, Fritz T, et al: Neuroinflammation leads to region-dependent alterations in astrocyte gap junction communication and hemichannel activity. J Neurosci 31: 14-425, 2011.

20. Stoimenov I and Helleday T: PCNA on the crossroad of cancer Biochem Soc Trans 37: 605-613, 2009.

21. Chiara AD, Pederzoli-Ribeil M, Burgel PR, et al: Targeting cytosolic proliferating cell nuclear antigen in neutrophil-dominated inflammation. Front Immunol 3: 311, 2012.
22. Strzalka W and Ziemienowicz A: Proliferating cell nuclear antigen (PCNA): a key factor in DNA replication and cell cycle regulation. Ann Bot 107: 1127-1140, 2011.

23. de Sousa Abreu R, Penalva LO, Marcotte EM, et al: Global signatures of protein and mRNA expression levels. Mol Biosyst 5: 1512-1526, 2009.

24. Ryten M, Dunn PM, Neary JT and Burnstock G: ATP regulates the differentiation of mammalian skeletal muscle by activation of a P2X5 receptor on satellite cells. J Cell Biol 158: 345-355, 2002.

25. Ryten M, Yang SY, Dunn PM, et al: Purinoceptor expression in regenerating skeletal muscle in the mdx mouse model of muscular dystrophy and in satellite cell cultures. FASEB J 18: 1404-1406, 2004.

26. Bao BA, Lai CP, Naus CC and Morgan JR: Pannexin1 drives multicellular aggregate compaction via a signaling cascade that remodels the actin cytoskeleton. J Biol Chem 287: 8407-8416, 2012.

27. McKuen MJ, Dahl G and Fields KA: Assessing a potential role of host Pannexin 1 during Chlamydia trachomatis infection. PLoS One 8: e63732, 2013.

28. Wang L, Zhu R, Huang Z, et al: Lipopolysaccharide-induced toll-like receptor 4 signaling in cancer cells promotes cell survival and proliferation in hepatocellular carcinoma. Dig Dis Sci 58: 2223-2236, 2013

29. Chen YH, Wang CC, Xiao X, et al: Multidrug resistance-associated protein 1 decreases the concentrations of antiepileptic drugs in cortical extracellular fluid in amygdale kindling rats. Acta Pharmacol Sin 34: 473-479, 2013.

30. Tietje K, Rivera-Ingraham G, Petters C, et al: Reporter dyes demonstrate functional expression of multidrug resistance proteins in the marine flatworm Macrostomum lignano: the sponge-derived dye Ageladine A is not a substrate of these transporters. Mar Drugs 11: 3951-3969, 2013.

31. Furugen A, Yamaguchi H, Tanaka N, et al: Contribution of multidrug resistance-associated proteins (MRPs) to the release of prostanoids from A549 cells. Prostaglandins Other Lipid Mediat 106: 37-44, 2013.

32. Wu J, Huang W and He Z: Dendrimers as carriers for siRNA delivery and gene silencing: a review. ScientificWorldJournal 29: 630654, 2013.

33. Wilson RC and Doudna JA: Molecular mechanisms of RNA interference. Annu Rev Biophys 42: 217-239, 2013.

34. Xu XW, Ding BW, Zhu CR, et al: PU.1-silenced dendritic cells prolong allograft survival in rats receiving intestinal transplantation. World J Gastroenterol 19(43): 7766-7771, 2013.

35. Kang ZH, Wang CY, Zhang WL, et al: Histone deacetylase HDAC4 promotes gastric cancer SGC-7901 cells progression via p21 repression. PLoS One 9: e98894, 2014.

36. Zhou M, Zhou L, Zheng L, et al: miR-365 Promotes Cutaneous Squamous Cell Carcinoma (CSCC) through Targeting Nuclear Factor I/B (NFIB). PLoS One 9: e100620, 2014. 